M. Schlosser $\cdot$ K. Koczwara $\cdot$ H. Kenk $\cdot$ M. Strebelow $\cdot$

I. Rjasanowski $\cdot$ R. Wassmuth $\cdot$ P. Achenbach $\cdot$

A.-G. Ziegler $\cdot$ E. Bonifacio

\title{
In insulin-autoantibody-positive children from the general population, antibody affinity identifies those at high and low risk
}

Received: 1 March 2005 / Accepted: 10 May 2005 / Published online: 12 July 2005

(C) Springer-Verlag 2005

\begin{abstract}
Aims/hypothesis: Insulin autoantibodies (IAA) precede and predict the onset of type 1 diabetes, but not all children with IAA develop the disease. In affected families, IAA affinity can identify IAA-positive children who are more likely to progress to diabetes. The purpose of this study was to determine whether affinity is a useful marker to stratify type 1 diabetes risk in IAA-positive children from the general population. Methods: IAA affinity was determined by competitive binding to ${ }^{125} \mathrm{I}$ insulin with increasing concentrations of cold insulin and with cold proinsulin in sera from 46 IAA-positive children identified in the Karlsburg Type 1 Diabetes Risk Study of a Normal Schoolchild Population in north-eastern Germany. Results: IAA affinity ranged between $5 \times 10^{6}$ and $1.2 \times 10^{11} \mathrm{l} / \mathrm{mol}$. IAA affinity was higher in 24 children who developed multiple islet autoantibodies or diabetes
\end{abstract}

M. Schlosser · H. Kenk

Institute of Pathophysiology,

University of Greifswald,

Karlsburg, Germany

K. Koczwara · P. Achenbach · A.-G. Ziegler · E. Bonifacio $(\square)$ Diabetes Research Institute,

Koelner Platz 1,

80804 Munich, Germany

e-mail: Ezio.Bonifacio@1rz.uni-muenchen.de

Tel.: +49-89-30793119

Fax: $+49-89-3081733$

M. Strebelow

LAD Hennigsdorf,

Branch Karlsburg,

Karlsburg, Germany

I. Rjasanowski

Center of Diabetes and Metabolic Disorders,

Karlsburg, Germany

R. Wassmuth

Institute for Transplantation Diagnostics

and Cell Therapeutics, Düsseldorf University,

Düsseldorf, Germany (median $3.5 \times 10^{9} \mathrm{l} / \mathrm{mol}$; interquartile range [IQR] $2.1 \times 10^{9}$ to $2.1 \times 10^{10} \mathrm{l} / \mathrm{mol}$ ) than in 22 children who did not develop multiple islet autoantibodies or diabetes (median $1.3 \times 10^{8}$ $1 / \mathrm{mol}$; IQR $3.8 \times 10^{7}$ to $\left.7.2 \times 10^{8} 1 / \mathrm{mol} ; p<0.0001\right)$. Using a threshold of $\geq 10^{9} 1 / \mathrm{mol}, 22$ of the 24 children who developed multiple islet autoantibodies or diabetes were correctly identified by high-affinity IAA and 18 of 22 who did not develop multiple islet autoantibodies or diabetes were correctly identified by low-affinity IAA. IAA affinity was significantly higher in samples with proinsulin reactive IAA $(p<0.0001)$. Conclusions/interpretation: IAA affinity measurement provides robust identification of IAA associated with high diabetes risk.

Keywords Antibody affinity - Insulin autoantibodies · Prediction · Proinsulin · Type 1 diabetes

Abbreviations IAA: insulin autoantibodies - IA-2: insulinoma antigen-2 IQR: interquartile range

\section{Introduction}

Autoantibodies against islet beta cell antigens can appear early in life and predict the development of type 1 diabetes [1-5]. Autoantibodies against insulin (IAA) are often the first autoantibodies that are detected in childhood. Some, but not all, children who have IAA develop antibodies to multiple beta cell antigens including GAD and insulinoma antigen-2 (IA-2) [1, 2, 5]. Children who do not develop autoantibodies to multiple beta cell antigens rarely progress to clinical type 1 diabetes during childhood [6]. For early risk assessment, therefore, it would be useful to identify IAA-positive children who will develop other islet autoantibodies. We showed that high IAA affinity and proinsulin reactivity were characteristics of IAA in first-degree relatives who developed further islet autoantibodies and type 1 diabetes [7]. The purpose of this study was to extend these findings to children from the general population. 


\section{Subjects, materials and methods}

Subjects and samples Samples were obtained from the Karlsburg Type 1 Diabetes Risk Study of a Normal Schoolchild Population [8, 9]. A total of 11840 children aged 6-17 years were screened for IAA using the polyethylene glycol IAA method, as well as for GAD, IA-2 and islet cell antibodies. For the present study, children with antibody levels above the 99th percentile were invited to provide a follow-up sample, which was obtained in 108 of the 158 initial IAA-positive children (median period of follow-up since the initial samples, 23 weeks; interquartile range [IQR] 16-38 weeks). Of these 108 follow-up samples, 54 were found to be positive in the polyethylene glycol IAA assay and the micro-IAA assay and were considered for IAA affinity measurement. In 46 of these 54 children, the sample volume was large enough to conduct IAA affinity measurement. Children had a median age of 10 years (IQR 8-12 years), 26 were male, and none had a first-degree relative with type 1 diabetes. Twenty-four had or developed antibodies to GAD and/or IA-2 $(n=23)$ and/or developed diabetes $(n=10), 21$ have remained positive only for IAA, and one was IAA-negative on further follow-up. All families of children provided written informed consent and the study was approved by the ethical committee of the Ernst-Moritz-Arndt Univer- sity Greifswald and by the Ministry of Culture and Education of Mecklenburg-Vorpommern.

IAA affinity and epitope analysis IAA affinity was measured in blinded fashion on coded sera by competitive inhibition in a protein $\mathrm{A} / \mathrm{G}$ radiobinding assay, as previously described [7]. Antibody binding to 7.85 fmol of human Tyr ${ }^{14} \mathrm{~A}\left[{ }^{125} \mathrm{I}\right]$ insulin (Aventis, Frankfurt, Germany; specific activity $370 \mathrm{MBq} / \mathrm{g} ; 0.128 \mathrm{nmol} / \mathrm{l}$ ) was competed with five increasing concentrations of unlabelled human insulin (Insuman Rapid; Aventis; 5.6 $\times 10^{-6}, 2.8 \times 10^{-7}, 2.8 \times 10^{-8}$, $\left.1.7 \times 10^{-9}, 5.5 \times 10^{-11} \mathrm{~mol} / \mathrm{l}\right)$ in a total volume of $55 \mu \mathrm{l}$ per tube. Duplicate tubes, each containing $5 \mu$ of serum, were used for each concentration of competing insulin. In order to determine the proinsulin reactivity of IAA, human proinsulin (Eli Lilly, Indianapolis, IN, USA; $1.0 \times 10^{-7} \mathrm{~mol} / \mathrm{l}$ ) was used for competition. $\mathrm{IC}_{50}$ and $K_{\mathrm{d}}$ values were calculated using the GraphPad Prism3 program (GraphPad Software, San Diego, CA, USA), and IAA affinity was expressed as reciprocal $K_{\mathrm{d}}$ values $(1 / \mathrm{mol})$ as previously described [7].

HLA typing HLA-DRB1 alleles were typed in 45 of the 46 children using PCR-amplified genomic DNA and nonradioactive sequence-specific oligonucleotide probes [10].
Fig. 1 IAA affinity in IAApositive school children in relation to IAA titre (a), the development of multiple islet autoantibodies or diabetes (b), proinsulin reactivity (c), and the presence of one or more HLA DRB $1 * 04$ alleles

(d). Filled circles $(\mathbf{a}, \mathbf{c}, \mathbf{d})$, children who developed multiple islet autoantibodies or diabetes; filled circles (b), children who developed diabetes. * $p<0.0001 ; \dagger p<0.0001 ; \# p=0.04$

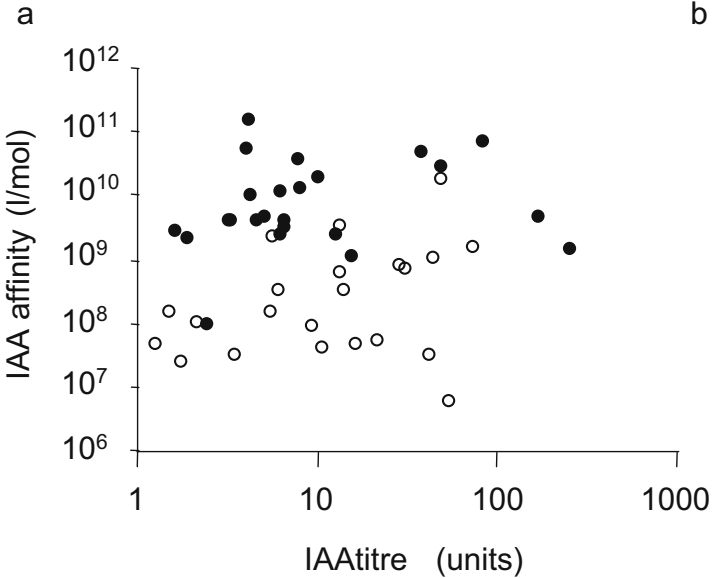

b
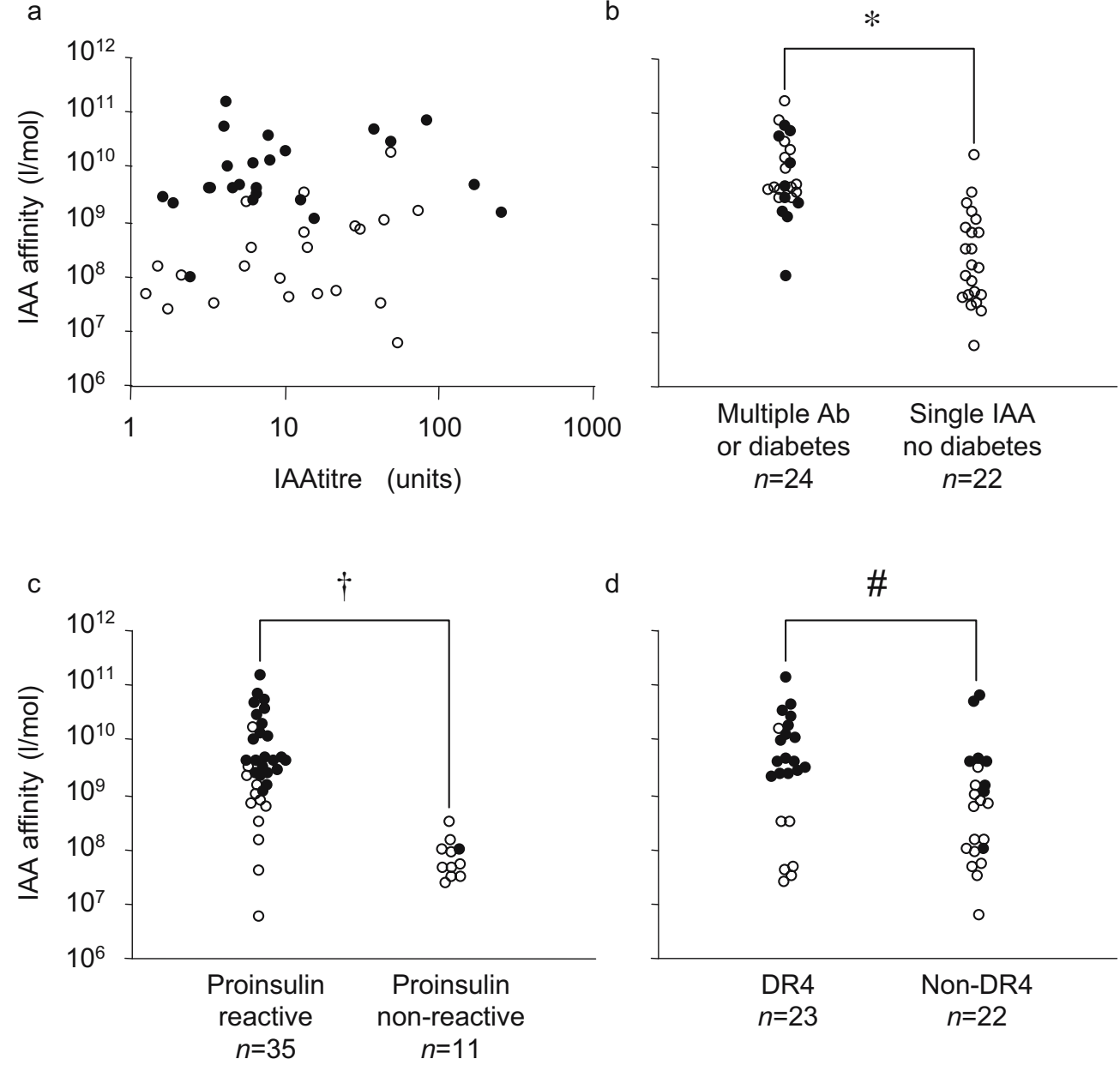

d

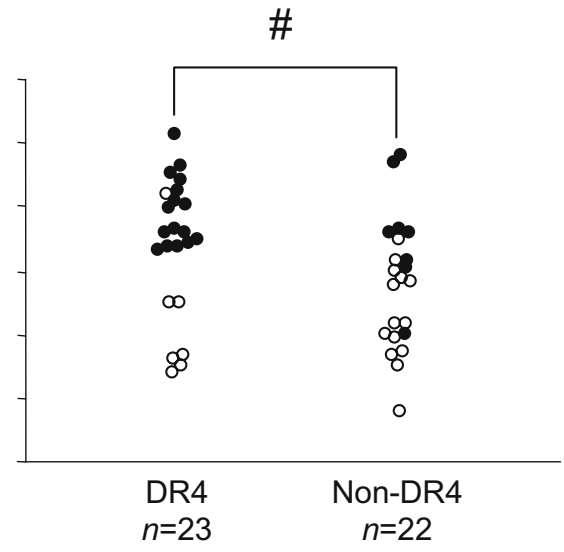


Statistical analysis Spearman's correlation was used to determine the correlation between IAA titre and IAA affinity. The Mann-Whitney $U$-test was used to compare IAA affinities between groups. Fisher's exact test was used to compare multiple antibody, type 1 diabetes, HLA risk and proinsulin reactivity between groups. For all analyses, a two-tailed $p$ value of 0.05 was considered significant. Statistical analyses were performed using the Statistical Package for the Social Sciences version 11.0 (SPSS, Chicago, IL, USA).

\section{Results and discussion}

IAA affinity ranged between $5 \times 10^{6}$ and $1.2 \times 10^{11} \mathrm{l} / \mathrm{mol}$ in the 46 IAA-positive samples. IAA affinity was not correlated with IAA titre $(r=0.08 ; p=0.58 ;$ Fig. 1a). The median IAA affinity was significantly higher in the 24 children who developed antibodies to GAD and/or IA-2 or diabetes (median IAA affinity $3.5 \times 10^{9} \mathrm{1} / \mathrm{mol}$; IQR $2.1 \times 10^{9}$ to $2.1 \times 10^{10} \mathrm{l} / \mathrm{mol}$ ) than in the 22 children who did not develop other islet autoantibodies or diabetes (median IAA affinity $1.3 \times 10^{8} \mathrm{l} / \mathrm{mol}$; IQR $3.8 \times 10^{7}$ to $7.2 \times$ $10^{8} \mathrm{l} / \mathrm{mol} ; p<0.0001$; Fig. $1 \mathrm{~b}$ ). As in findings in IAA-positive first-degree relatives [7], IAA affinity of $\geq 10^{9} 1 / \mathrm{mol}$ was a discriminatory threshold: $22(92 \%)$ of the 24 children who developed other islet autoantibodies or diabetes had IAA affinities $\geq 10^{9} 1 / \mathrm{mol}$, compared with only four $(18 \%)$ of 22 of the children who did not progress to multiple islet autoantibodies or diabetes $(p<0.0001)$.

IAA in 11 of the 46 samples could not be inhibited by cold proinsulin and were considered negative for proinsulin antibody. All 11 had an IAA affinity $<10^{9} 1 / \mathrm{mol}$ (median IAA affinity $4.4 \times 10^{7} \mathrm{l} / \mathrm{mol}$; IQR $2.8 \times 10^{7}$ to $8.7 \times 10^{7} \mathrm{l} / \mathrm{mol}$ ), and these samples had a lower IAA affinity than the 35 proinsulin-reactive samples (median IAA affinity $2.7 \times 10^{9} \mathrm{l} / \mathrm{mol}$; IQR $9.9 \times 10^{8}$ to $1.1 \times 10^{10}$; $p<0.0001$; Fig. 1c). IAA affinity was higher in children who had the HLA DRB1*04 allele, which confers susceptibility to type 1 diabetes (median IAA affinity $3.3 \times 10^{9}$ $1 / \mathrm{mol}$; IQR $2.5 \times 10^{8}$ to $1.3 \times 10^{10} \mathrm{l} / \mathrm{mol}$, vs median IAA affinity $6.1 \times 10^{8} \mathrm{l} / \mathrm{mol}$; IQR $8.1 \times 10^{7}$ to $2.8 \times 10^{9} \mathrm{l} / \mathrm{mol}$ in the remainder; $p=0.04$; Fig. $1 \mathrm{~d}$ ).

The findings in the IAA-positive children from the general population are consistent with those in IAApositive first-degree relatives. IAA affinity varied over nearly 5 logs. IAA that was associated with multiple autoantibody positivity and diabetes was of high affinity and proinsulin-reactive, whereas most children who had only IAA and did not develop multiple antibodies had lower affinity IAA and almost half had IAA that did not bind proinsulin. Also in both studies, the HLA DRB $1 * 04$ allele was more frequent in children who had multiple islet autoantibodies, and was therefore more frequent in children with high affinity. The findings are unlikely to be an artefact resulting from measuring weak or non-reproducible IAA-positive samples, since all IAA-positive children selected for study had IAA confirmed in two samples using two different IAA assays.
In this study, the measurement of IAA affinity was performed using only five concentrations of cold insulin as competitor, and was achieved with $60 \mu \mathrm{l}$ of serum. Affinity values obtained in this manner on coded samples could identify high-risk (developed multiple antibodies or diabetes) and low-risk (remained IAA-positive only and non-diabetic) children. Moreover, $44 \%$ of confirmed IAApositive children had a low-risk IAA phenotype. Thus, we have identified a relatively simple and robust method that will be useful for risk assessment in prospective studies in infants.

Acknowledgements The study was supported by the Federal Ministry of Research and Technology (Grant 07NBL02/D4), the Ministry of Culture and Education of Mecklenburg-Vorpommern (Grant EMAU16/1995), the German Diabetes Foundation (Grant $133 / 05 / 03)$, the Else Kröner-Fresenius Foundation, the Division of Employment Stralsund and the Deutsche Forschungsgemeinschaft (Grant SFB263 and Grant ZI 310/12-6).

Duality of Interest

The authors are not aware of any conflicts of interest in this study.

\section{References}

1. Ziegler AG, Hummel M, Schenker M, Bonifacio E (1999) Autoantibody appearance and risk for development of childhood diabetes in offspring of parents with type 1 diabetes: the 2-year analysis of the German BABYDIAB study. Diabetes 48:460-468

2. Colman PG, Steele C, Couper JJ et al (2000) Islet autoimmunity in infants with a type I diabetic relative is common but is frequently restricted to one autoantibody. Diabetologia 43:203209

3. Yu L, Robles DT, Abiru N et al (2000) Early expression of antiinsulin autoantibodies of humans and the NOD mouse: evidence for early determination of subsequent diabetes. Proc Natl Acad Sci U S A 97:1701-1706

4. Atkinson MA, Eisenbarth GS (2001) Type 1 diabetes: new perspectives on disease pathogenesis and treatment. Lancet 358:221-229

5. Kimpimaki T, Kulmala P, Savola K et al (2002) Natural history of beta-cell autoimmunity in young children with increased genetic susceptibility to type 1 diabetes recruited from the general population. J Clin Endocrinol Metab 87:4572-4579

6. Hummel M, Bonifacio E, Schmid S, Walter M, Knopff A, Ziegler AG (2004) Brief communication: early appearance of islet autoantibodies predicts childhood type 1 diabetes in offspring of diabetic parents. Ann Intern Med 140:882-886

7. Achenbach P, Koczwara K, Knopff A, Naserke H, Ziegler AG, Bonifacio E (2004) Mature high-affinity immune responses to (pro)insulin anticipate the autoimmune cascade that leads to type 1 diabetes. J Clin Invest 114:589-597

8. Strebelow M, Schlosser M, Ziegler B, Rjasanowski I, Ziegler M (1999) Karlsburg type 1 diabetes risk study of a general population: frequencies and interactions of the four major type 1 diabetes-associated autoantibodies studied in 9,419 schoolchildren. Diabetologia 42:661-670

9. Schlosser M, Strebelow M, Rjasanowski I, Kerner W, Wassmuth R, Ziegler M (2004) Prevalence of diabetes-associated autoantibodies in schoolchildren: the Karlsburg type 1 diabetes risk study. Ann NY Acad Sci 1037:114-117

10. Schlosser M, Strebelow M, Wassmuth R et al (2002) The Karlsburg type 1 diabetes risk study of a normal schoolchild population: association of beta-cell autoantibodies and human leukocyte antigen-DQB1 alleles in antibody-positive individuals. J Clin Endocrinol Metab 87:2254-2261 